\title{
Automatic Brain Tissues Segmentation based on Self Initializing K-Means Clustering Technique
}

\author{
Kalaiselvi T \\ Assistant Professor, Department of Computer Science and Applications, \\ The Gandhigram Rural Institute - Deemed University, Tamil Nadu, India \\ E-mail: kalaiselvi.gri@gmail.com \\ Kalaichelvi N, Sriramakrishnan P \\ Research Scholar, Department of Computer Science and Applications, \\ The Gandhigram Rural Institute - Deemed University, Tamil Nadu, India \\ E-mail: chelvi.kalai7@gmail.com, sriram0210@gmail.com
}

Received: 30 March 2017; Accepted: 05 June 2017; Published: 08 November 2017

\begin{abstract}
This paper proposed a self-initialization process to K-Means method for automatic segmentation of human brain Magnetic Resonance Image (MRI) scans. $\mathrm{K}-\mathrm{Means}$ clustering method is an iterative approach and the initialization process is usually done either manually or randomly. In this work, a method has been proposed to make use of the histogram of the gray scale MRI brain images to automatically initialize the K-means clustering algorithm. This is done by taking the number of main peaks as well as their values as number of clusters and their initial centroids respectively. This makes the algorithm faster by reducing the number of iterations in segmenting the MRI image. The proposed method is named as Histogram Based Self Initializing K-Means (HBSIKM) method. Experiments were done with the MRI brain volumes available from Internet Brain Segmentation Repository (IBSR). Similarity validation was done by Dice coefficient with the available gold standards from the IBSR website. The performance of the proposed method is compared with the traditional KMeans method. For the IBSR volumes, the proposed method yields 3 to 4 times faster results and higher dice value than traditional K-Means method.
\end{abstract}

Index Terms-K-Means, self-initialization, histogram, bounding box, MRI brain, MRI scans.

\section{INTRODUCTION}

Image segmentation is an important image processing technique that partitions the image into number of homogeneous regions based on the characteristics of the pixels [1]. Segmentation depends on various features like regions, edges, color, and texture etc. of the image. Thus the segmentation techniques are categorized into threshold based, edge based and region based segmentation. Threshold based image segmentation allocates the pixels into categories according to the range of pixel values. Edge based image segmentation groups the pixels into edge or non- edge depending on the edge filter's output, applied to the image. Region based image segmentation algorithms operate pixels iteratively by grouping neighbors with similar values [2].

Segmentation and clustering techniques can be applied in various fields in the medical domain to analyze and identify the abnormalities present in organs like human heart, bone of knee and brain [3] [4]. The abnormalities form in brain due to any of the disorders like tumors, seizure, stroke, trauma and head injury etc. The diagnostic process based on manual segmentation by medical experts is time consuming. Hence Computer Aided Diagnostics (CAD) systems are aimed in medical field at present [5].

There are various modalities available for imaging organs, some of which are computed tomography (CT), magnetic resonance imaging (MRI), electroencephalography (EEG), magneto encephalography (MEG) and positron emission tomography (PET). MRI uses radio waves in a strong magnetic field to create detailed images of organs and tissues. MRI has been proven to be highly effective in diagnosing normal and diseased tissues of the body and produces more accurate results [6].

The MR images provide a large amount of data with high quality when compared with other modalities data. It provides good contrast between the soft tissues of body. Thus the segmentation in MRI results better classification of brain tissues, its surrounding fluid and background. The classification of these tissues will help to analyze and study the anatomy and functions of brain [7]. Algorithms are derived to segment different types of tissues in organs like gray matter (GM), white matter (WM), cerebrospinal fluid (CSF) and tumor region (if it is an abnormal brain) in MRI brain image [8].

K-Means clustering is one of the hard segmentation algorithms used in various applications. But the main limitation of this algorithm is the selection of number of clusters and initial centroids to segment the given input data. In digital image processing, the selection of initial centroids can be done automatically using many ways. 
The proposed method uses image histogram to get the initial centroids and the number of clusters automatically and named as Histogram Based Self Initializing K-Means (HBSIKM) method. Experiments were done with the MRI brain volumes available from Internet Brain Segmentation Repository (IBSR). Similarity validation was done by Dice coefficient with the available gold standards from the IBSR website. The performance of the proposed method is compared with the traditional $\mathrm{K}$ Means method. The similarity results show that the proposed method runs efficiently in segmenting the tissue regions with minimum number of iterations and also with the self-initialized centroids of MRI brain images. For the IBSR volumes, the proposed method yields 3 to 4 times faster results and higher dice value than traditional $\mathrm{K}$ Means method.

In this paper, section II contains the related works done and section III contains the methodology. The results are discussed in section IV and conclusion is given in section V.

\section{RELATED WORK}

Kaufman and Rousseeuw have proposed an algorithm to initialize the K-Means segmentation by manually selecting the K-value [9] [10]. The first centroid is selected as the most centrally located instance and the other centroids are taken as the higher numbered instances among the rest of data in the database. The limitation is the manual initialization of the number of clusters and their centroids. Bapusaheb and Bansode have developed Pillar algorithm in which the first centroid is taken as the grand mean of the dataset [11]. The data point with a maximum distance from the previous centroid is taken as the next centroid. Thus the other centroids are found out in an iterative manner by calculating the distance metrics. But it has the limitation of adjusting the characteristics of data distribution in dataset, in order to set up the appropriate parameters for outlier detection mechanism.

Tian et al., have proposed a method to automatically initialize the K-Means clustering algorithm based on histogram of the input dataset [12]. In this algorithm, all the local maxima in the histogram are taken and the global maxima among them are selected as the first centroid. And the remaining centroids are taken iteratively with the help of a maximized distance measure (DM). The DM is the product of height of local maxima and the distance from previous centroids. Here the limitation is manual selection of centroids and $\mathrm{K}$ (no. of classes) value. Mohamed et al., have proposed an EIKmeans algorithm that made improvement over the $\mathrm{K}$ Means segmentation algorithm by introducing a novel density function based on k-nearest neighbor method [13] By this definition the noise and other outliers that affect Kmeans strongly are obtained along with the initial centroids and number of clusters automatically.

Raed has done the initialization of centroids based on calculating the Euclidean distance and Manhattan distance between the data points and centroids [14]. But this process starts with randomization of the centroids. Kalaiselvi and Somasundaram have proposed an algorithm called FCM-Expert where the initialization to four clusters is done manually from the knowledge of the MRI brain scan images [5]. Here the peak values from the histogram are taken in to consideration. The background intensity is taken as 0 and white matter as 255 and the peak values 85 and 170 are taken as centroids to cerebrospinal liquid and gray matter respectively. The fixed values initialization is done with knowledge based technique.

In the proposed method, the initialization is done automatically with the help of histogram of the gray scale MRI brain image. The middle slice in an MRI volume is considered to have all the tissue regions thus its histogram is chosen to get the initial $\mathrm{K}$ and their centroid values. The number of peaks and their values are taken to initialize the number of classes and the centroids in the K-Means algorithm. The final centroids of each slice are used to initialize the adjacent slice's K-Means segmentation process. The pre-processes involved in the proposed method are brain portion extraction and bounding box cropping to remove the unwanted background from the input image. Finally, the performance evaluation is done by comparing the results with the ground truth values available from IBSR datasets.

\section{Methodology}

K-Means segmentation is an unsupervised classification algorithm that needs the manual initialization of the number of classes as well as randomization of initial centroids of each class. The initial cluster is created by associating each pixel in the image to the given nearest centroid, then the mean values of the elements in the clusters are computed and the centroids are replaced by them. These steps are done iteratively until there is no more in centroid [15]. The traditional K-Means algorithm is given below.

\section{Algorithm 1: K-Means clustering}

Step 1: Set $\mathrm{K}$ and $\mathrm{C}$, the number of clusters required and initial centroids randomly.

Step 2: Allocate each pixel to the nearest class. This is done by minimizing the objective function $\mathrm{J}$ as given below.

$$
J=\sum_{j=1}^{K} \sum_{i=1}^{n}\left\|x_{i}^{(j)}-c_{j}\right\|^{2}
$$

where, $\left\|x_{i}^{(j)}-c_{j}\right\|^{2}$ is a chosen distance measure between a data point $x_{i}^{(j)}$ and the cluster center $c_{j}$. It is an indicator of the distance of the $n$ data points from their respective cluster centers.

Step 3: Compute mean value for each cluster and replace the new centroid as the mean value.

Step 4: Repeat steps 2 and 3 until no more new centroids are created. 
The main aim of the Histogram Based Self Initializing K-Means (HBSIKM) method is to fully automate the KMeans clustering algorithm using the normalized histogram of the MRI gray scale. Fig. 1 shows the flow chart of proposed method. The three main processes of the proposed method are brain portion extraction, bounding box cropping and histogram based centroids initialization. Brain portion extraction is done by BEM algorithm for each image. As a result the brain region alone is extracted [6]. The bounding box technique is applied on this image to ignore unwanted background and crop the AOI that is the brain portion alone. Then the histogram is taken for the middle slice of each dataset since the middle slice is considered to have all the tissue regions clearly. This is done in order to get the exact number of clusters and their centroids in the MRI brain dataset. Gaussian smoothening is applied over the histogram to get distinct peaks. The automatic initialization of the number of clusters $(\mathrm{K})$ is done with the number of peaks and initial centroids with the peak values of the normalized histogram. With these initial $\mathrm{K}$ and $\mathrm{C}$ values, the $\mathrm{K}-\mathrm{Means}$ segmentation is performed on each slice in the dataset. For each time the final centroid of the current slice is taken as the initial centroid for the next slice in the dataset. These three processes support to enhance the performance of traditional K-Means method by reducing the number of iterations in the segmentation of each image and thus the time too.

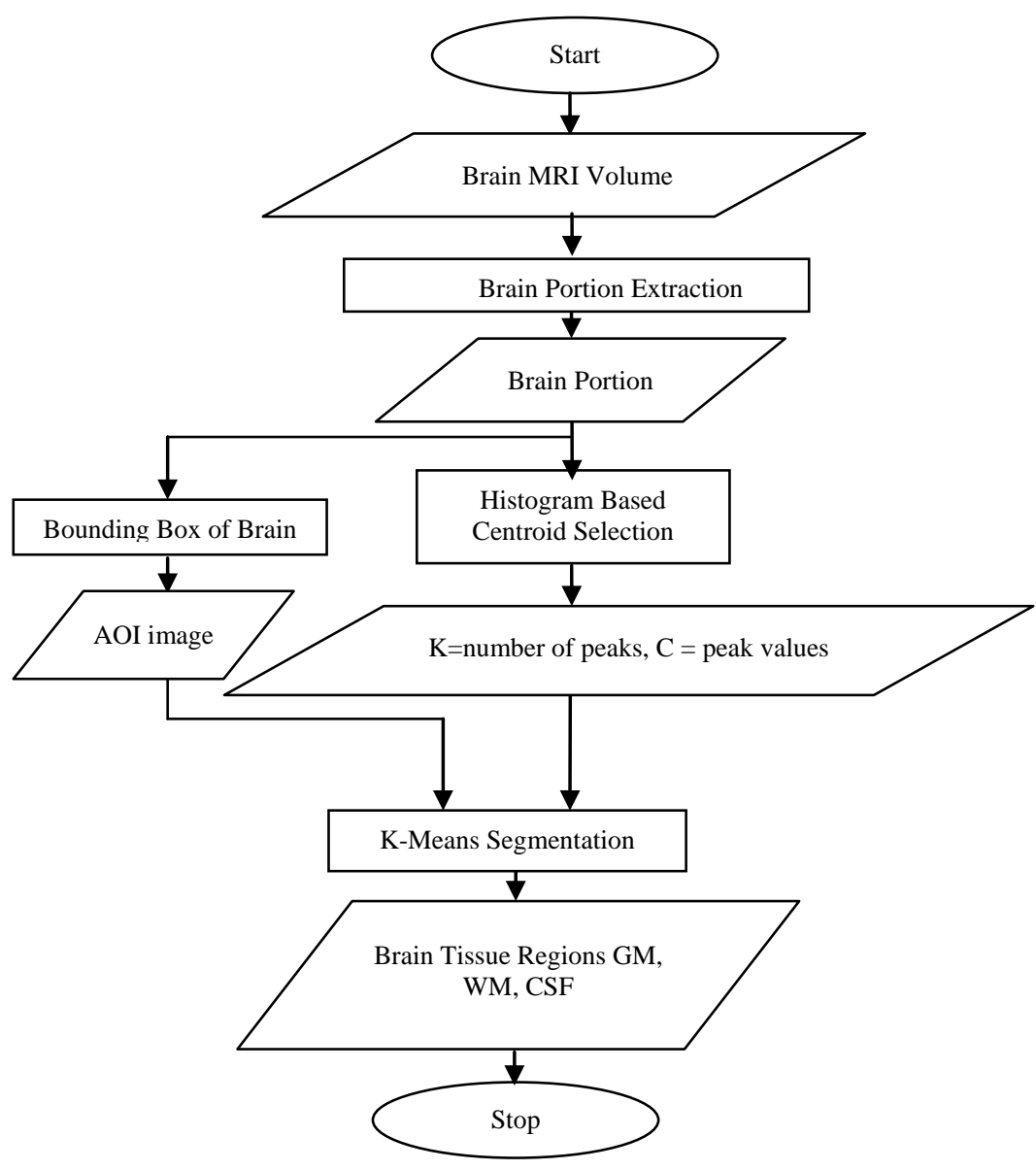

Fig.1. Flow chart of the proposed method

Algorithm 2: HBSIKM

Input : MRI volume

Step 1: Apply BEM algorithm to extract brain portion.

Step 2: The image histogram of the middle slice generated.

Step 3: Apply Gaussian filtering on the histogram in order to smoothen the histogram that yields distinct peaks. The Gaussian distribution is

$$
G(x)=\frac{1}{\sigma \sqrt{2} \pi} e^{-\frac{(x-\mu)^{2}}{2 \sigma^{2}}}
$$

Step 4: Find the number of peaks $K$ and peak values $C$. Step 5: Initiate the number of clusters $\mathrm{K}$ by $\mathrm{m}$ and the initial centroids $c_{j}$ by a where $\mathrm{j}=1,2 \ldots \mathrm{K}$. for the middle slice in the dataset.

Step 6: Select the AOI using bounding box cropping process.

Step 7: Apply K-Means segmentation for the AOI.

Step 8: Use the final centroids of the previous slice to initialize the centroids for current slice

Step 9: Repeat the previous two steps for the remaining slices in the dataset. 


\section{A. Brain Portion Extraction}

The MRI brain image contains three portions skull, scalp and the soft brain inside. Here the AOI is the brain portion where the three main tissues GM, WM and CSF reside. Thus the first step is to extract the region of interest i.e. the brain portion from the skull and scalp. For this the BEM is applied. This is a fully automatic twostage brain extraction method that extracts the brain

portion from the MRI images using feature extraction in first stage and morphological and connected component operations in the second stage to produce the fine brain portion. A sample coronal MRI image is shown in Fig.2 (a) and its extracted brain portion is given in Fig.2 (b). This sample image corresponds to the middle slice of 205_3 voulme from IBSR20 dataset.

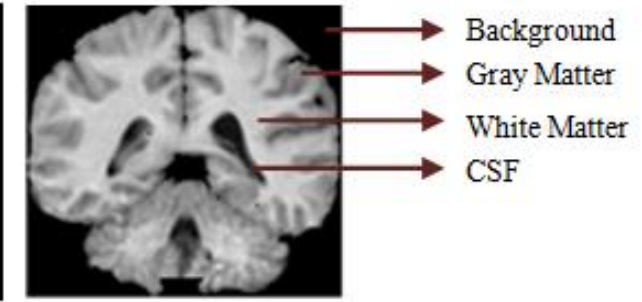

Fig.2. a) Sample MRI head scan b) Extracted brain after BEM c) AOI after bounding box cropping

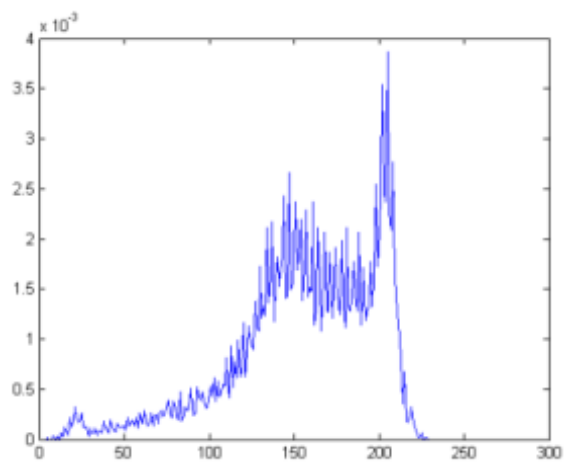

(a)

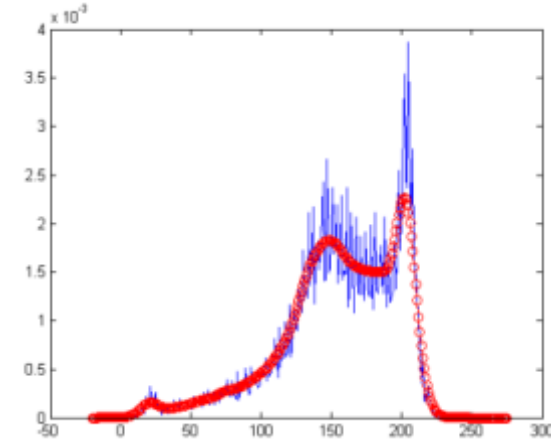

(b)

Fig.3. (a) Histogram (b) smoothed histogram

\section{B. Bounding Box Cropping}

The bounding box technique is applied on the resultant image of the previous step in order to extract the brain portion alone as the AOI by cropping the unwanted background. This is done in following steps.

\section{Algorithm 3: Bounding Box Cropping}

Step 1: Scan the input image row wise from top to bottom until a row with a data $>0$ is reached. This row is taken as rowmin.

Step 2: Scan the image row wise from bottom to top until a row with a data $>0$ is reached. This row is taken as rowmax.

Step 3: Scan column wise from left to right until a column with data $>0$ is reached. This column is taken as colmin.

Step 4: Scan column wise from right to left until a column with data $>0$ is reached. This column is taken as the colmax.

This bounding box algorithm is applicable for MRI brain images since the background is set to zero by BEM. The AOI of the image lies within the rowmin, colmin and rowmax, colmax coordinates of the image. The cropped AOI image with the indication of BG, CSF, GM and WM is shown in Fig.2(c).

\section{Histogram Based Centroid Selection}

Image histogram is the graphical representation of the intensity distribution over the pixels of the image. The histogram of the middle slice is taken in order to get the number of tissue regions present in the brain image and also contains the most frequent intensity value in each region. Since the middle slice is considered to have all the tissue regions, it is selected for the histogram based centroid selection. The low pass Gaussian smoothening filter is applied on the histogram as given in equation (2) in order to get the distinct peaks. The histogram produces peaks corresponding to the number of regions present in the brain image. Since the brain has three main regions CSF, GM and WM, the histogram produces three main peaks that is taken as the number of centroids $K$, and the corresponding peak values for $c_{j}$ (centroid of each region) where $\mathrm{j}=1,2,3 \ldots \mathrm{K}$. The histogram and the smoothened histogram with distinct peaks for the middle slice of IBSR 205_3 dataset are shown in Fig.3 (a) and Fig.3 (b) respectively.

\section{K-Means segmentation}

$\mathrm{K}-\mathrm{Means}$ segmentation in the proposed method begins with the initial $\mathrm{K}$ as the number of smoothened peaks and the centroids $c_{j}$ as the peak values in the histogram, taken 
for the AOI portion of the middle slice. Because of the adjacency nature among the slices of the MRI volume, the final centroids of each slice is taken as the initial centroids for the adjacent images. The K-Means clustering is performed as given in Algorithm 1.

\section{E. Evaluation Metrics}

The Dice coefficient is an index to measure the similarity of the segmentation. It is calculated as

$$
D=\frac{2|A \cap B|}{|A|+|B|}
$$

where, $\mathrm{A}$ and $\mathrm{B}$ are the pixel count of the respective regions in ground truth image and segmented image. The performance evaluation of the proposed method is done by calculating Dice coefficient (D) and compared with that of traditional K-means clustering algorithm.

\section{RESULTS AND DISCUSSIONS}

The experiments were carried out on 20 and 18 Coronal T1 weighted IBSR datasets. Initially each slice is applied with the brain portion extraction algorithm in which the segmentation of brain portion is done in two stages. The first stage performs feature extraction where a rough brain portion is extracted with the help of run length identification for labeling the brain, scalp and background, skull and CSF. In the second stage a fine brain mask is created using morphological and largest connected component operations. In this stage, the adjacent slice's similarity property is used to select the proper brain regions. As a result the brain portions are extracted for each slice in the above said datasets.

The proposed HBSIKM and the traditional K-Means algorithms are applied on the resultant images of the BEM algorithm. From each dataset, the slices with the major brain portions were taken for the processes by leaving the end slices present in both ends of the dataset in order to avoid the wastage of time, since these slices contains less amount of brain information.

The histogram of the input brain portion is taken for the middle slice of the dataset, and Gaussian smoothening is applied on the histogram in order to get distinct peaks. From the smoothened histogram the number of centroids and the initial centroids are taken for further processing. Then the bounding box cropping is done on all the slices in order to avoid the unwanted background. With these initial values the K-means segmentation is performed on the all the slices from the beginning of the dataset except those slices with less brain portions by carrying the final centroids of one slice to the next slice's initial centroids.

As the result of above said segmentation processes, the three main tissues CSF, GM and WM of each slice are obtained. The respective sample images are shown in Fig. 4. Figure 4 contains the smoothened histogram with distinct peaks in column1, the original image, the CSF region, the GM region, the $\mathrm{WM}$ region and the triclustered region images in column 2, 3, 4, 5 and 6 respectively. The CSF region contains the sulcul CSF around the GM in the result of proposed method.
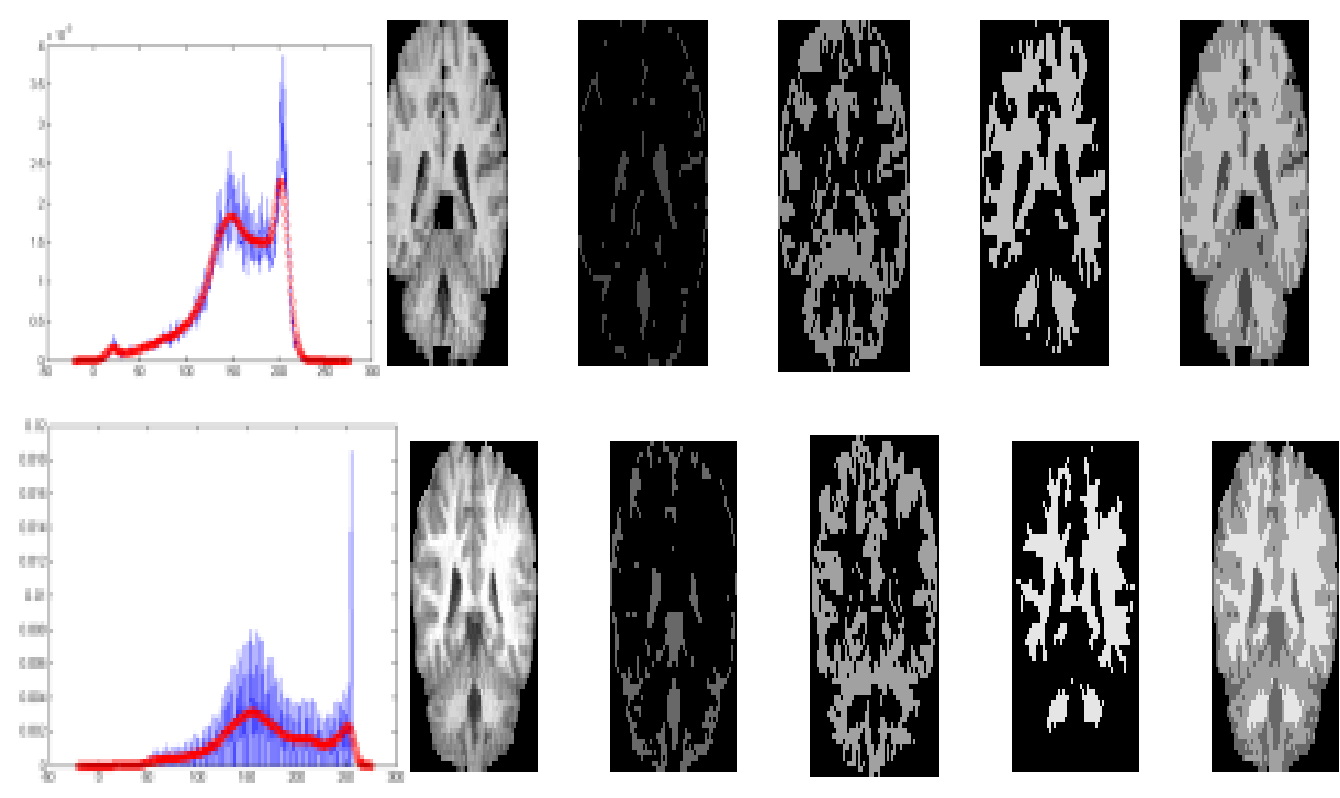

Fig.4. Results of proposed HBSIKM method Column 1: Smoothened histogram, Column 2: Original slices, Column 3: CSF region, Column 4: GM region, Column 5: WM region and Column 6: tri-clustered image. 


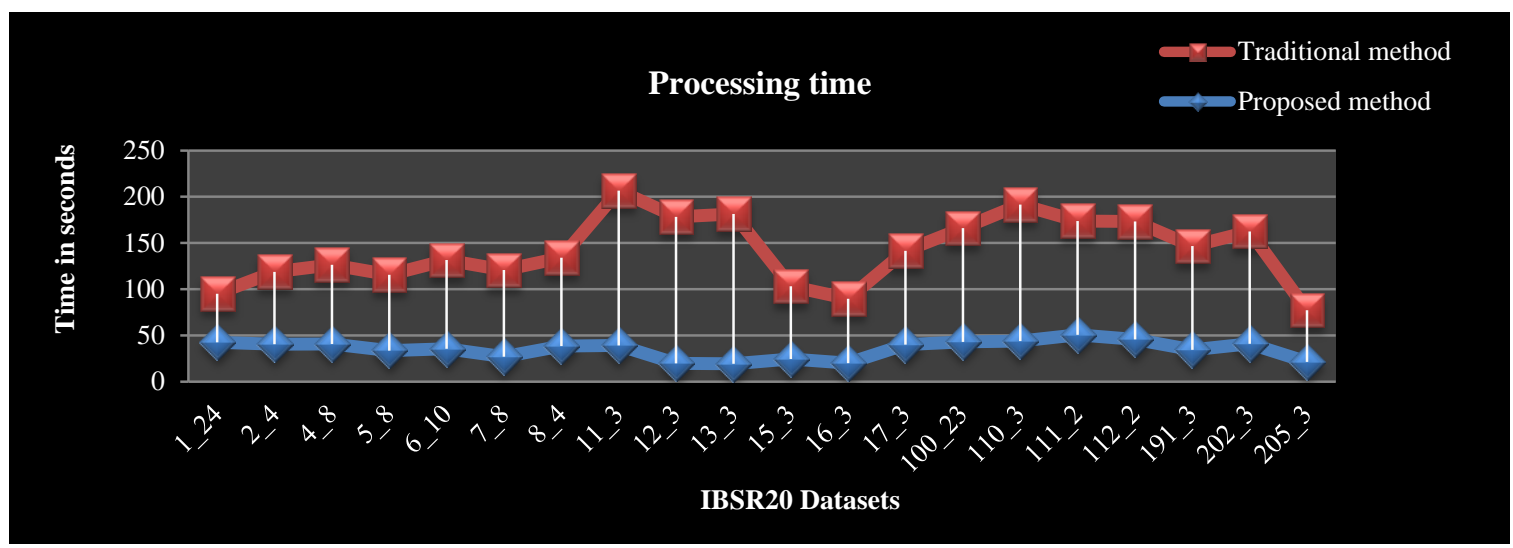

(a)

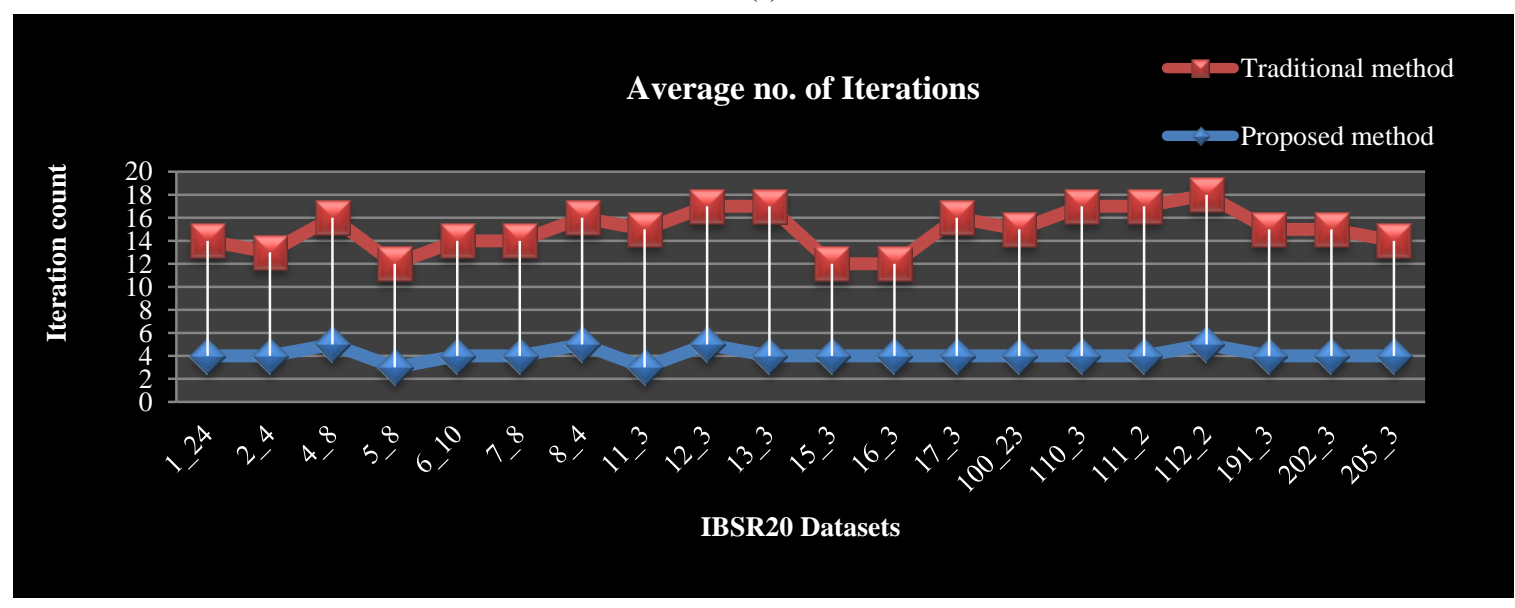

(b)

Fig.5. Performance comparison of traditional and proposed methods on IBSR20 volumes a) Processing time comparison b) Average iteration count comparison

In the proposed method, since the initialization is done automatically and the centroids are carried over to the adjacent slices to initialize their centroids, the number of iterations and the time taken to segment for each slice is reduced. The comparisons between the traditional as well as the proposed methods are shown in Fig 5, 6, 7 and 8.

The comparison between the traditional as well as the proposed algorithms in terms of average time taken and the number of iterations to segment per slice of each volume in IBSR20 datasets are shown in Fig.5 (a) and Fig.5 (b) respectively.
The comparison chart for average time taken per volume in IBSR18 is shown in Fig.6 (a). The comparison chart for average iteration count per slice of each volume in IBSR18 dataset is shown in Fig.6 (b). From Fig.5 and 6 our proposed HBSIKM algorithm is approximately 3 to 4 times faster than the traditional K-Means algorithm. A quantitative analysis is performed to examine the segmentation by calculating the Dice coefficient between the ground truth and the resultant images of traditional and proposed methods.

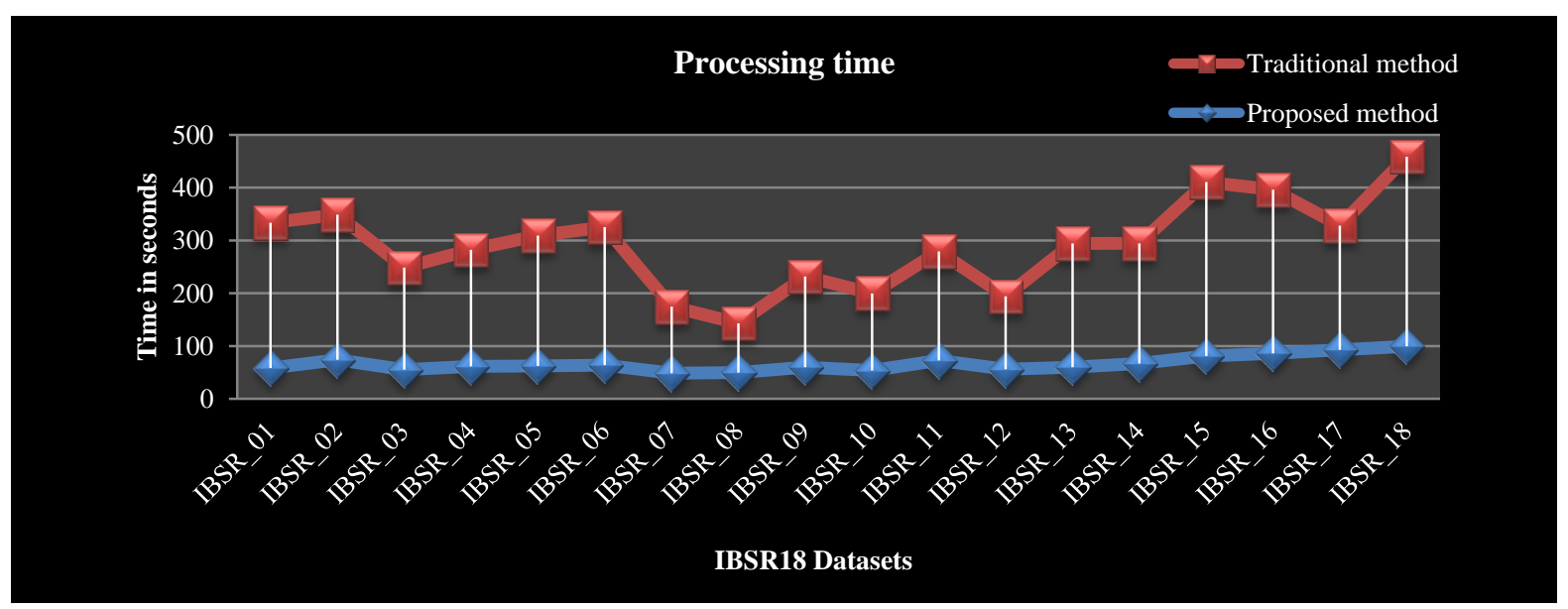

(a) 


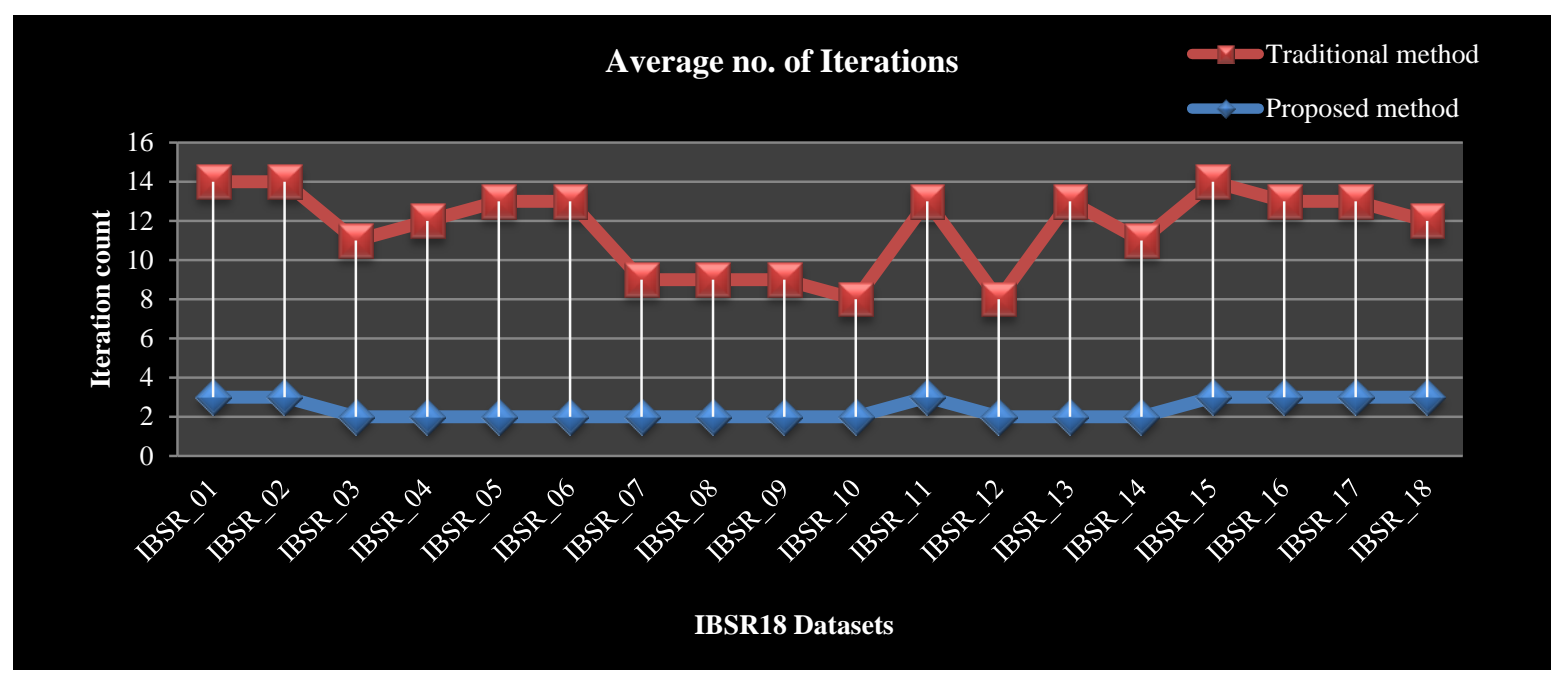

(b)

Fig.6. Performance comparison of traditional and proposed methods on IBSR18 volumes a) Processing time comparison b) Iteration count comparison

Table 1 shows the comparison between the average Dice coefficients of the results of HBSIKM and traditional K-Means for IBSR20 datasets. The final mean dice value of IBSR20 datasets is shown in Fig.7 as bar chart. Since the gold standard for these volumes consist SCSF, the similarity coefficients for CSF have increased.

Table 2 shows the comparison between the average Dice coefficients of the results of proposed and traditional K-means for IBSR18 dataset. The final mean dice value of IBSR 18 dataset is shown in Fig.8 as bar chart. Since the proposed method's CSF region includes the Sulcal Cerebrospinal Fluid (SCSF) in the surrounding, and it is not included in the corresponding IBSR gold standard images, the similarity coefficients for the corresponding segmentation results have got reduced for IBSR 18 dataset.

Table 1. Average Dice coefficient of each volume in IBSR20 dataset

\begin{tabular}{|c|c|c|c|c|c|c|}
\hline \multirow{2}{*}{ Volume } & \multicolumn{2}{|c|}{ Traditional K-Means } & \multicolumn{3}{c|}{ Proposed HBSIKM } \\
\cline { 2 - 7 } & CSF & GM & WM & CSF & GM & WM \\
\hline 1_24 & 0.1538 & 0.7086 & 0.8424 & 0.1463 & 0.7368 & 0.8466 \\
\hline 2_4 & 0.1385 & 0.6456 & 0.7559 & 0.0683 & 0.5929 & 0.7421 \\
\hline 4_8 & 0.1148 & 0.6644 & 0.7366 & 0.1888 & 0.6500 & 0.7704 \\
\hline 5_8 & 0.0667 & 0.6677 & 0.7845 & 0.1964 & 0.7235 & 0.8266 \\
\hline 6_10 & 0.0584 & 0.6710 & 0.7577 & 0.2657 & 0.7022 & 0.8219 \\
\hline 7_8 & 0.0667 & 0.7198 & 0.8355 & 0.1416 & 0.7336 & 0.8483 \\
\hline 8_4 & 0.1270 & 0.7160 & 0.8156 & 0.1693 & 0.7306 & 0.8368 \\
\hline 11_3 & 0.0333 & 0.7209 & 0.8335 & 0.2444 & 0.7565 & 0.8683 \\
\hline 12_3 & 0.0635 & 0.7158 & 0.8425 & 0.2595 & 0.7650 & 0.8868 \\
\hline 13_3 & 0.0952 & 0.7114 & 0.8447 & 0.0763 & 0.7190 & 0.8687 \\
\hline 15_3 & 0.0833 & 0.5955 & 0.6506 & 0.2203 & 0.6226 & 0.7107 \\
\hline 16_3 & 0.1500 & 0.6828 & 0.7477 & 0.1596 & 0.6790 & 0.7762 \\
\hline 17_3 & 0.1111 & 0.6912 & 0.8076 & 0.1738 & 0.6790 & 0.8337 \\
\hline 100_23 & 0.1587 & 0.7259 & 0.8303 & 0.1043 & 0.7490 & 0.8729 \\
\hline 110_3 & 0.1270 & 0.7477 & 0.8229 & 0.1681 & 0.7572 & 0.8457 \\
\hline 111_2 & 0.5542 & 0.1094 & 0.7004 & 0.1546 & 0.7561 & 0.8597 \\
\hline 112_2 & 0.1270 & 0.7419 & 0.8222 & 0.1546 & 0.7385 & 0.8246 \\
\hline 191_3 & 0.1111 & 0.7373 & 0.8437 & 0.1260 & 0.7531 & 0.8580 \\
\hline 202_3 & 0.0952 & 0.7300 & 0.8438 & 0.2604 & 0.7722 & 0.8698 \\
\hline Mean & $\mathbf{0 . 1 2 8 2}$ & $\mathbf{0 . 6 6 8 6}$ & $\mathbf{0 . 7 9 5 7}$ & $\mathbf{0 . 1 7 2 5}$ & $\mathbf{0 . 7 1 6 7}$ & $\mathbf{0 . 8 2 9 9}$ \\
\hline
\end{tabular}




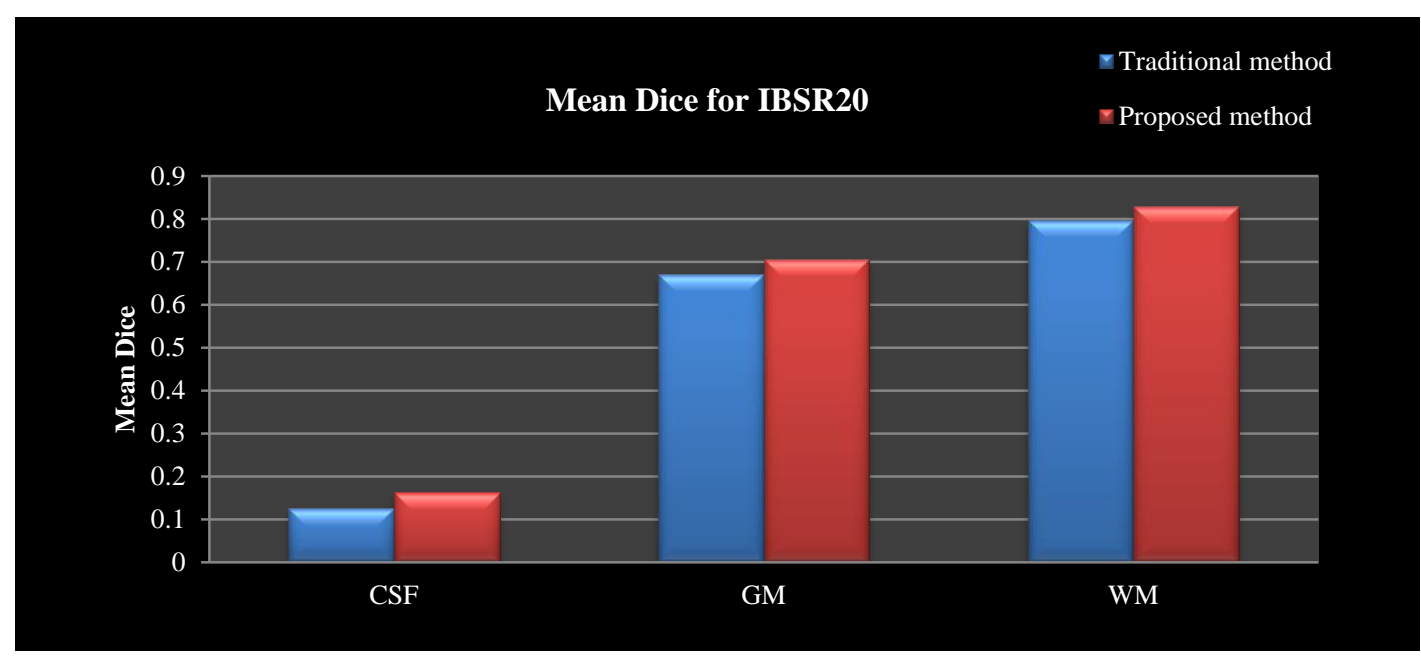

Fig.7. Mean Dice comparison between traditional and proposed HBSIKM for IBSR20 dataset

Table 2. Average Dice coefficient of each volume in IBSR18 dataset

\begin{tabular}{|c|c|c|c|c|c|c|}
\hline \multirow{2}{*}{ Volume } & \multicolumn{2}{|c|}{ Traditional K-Means } & \multicolumn{3}{c|}{ Proposed HBSIKM } \\
\cline { 2 - 7 } & CSF & GM & WM & CSF & GM & WM \\
\hline IBSR_01 & 0.5294 & 0.7572 & 0.7966 & 0.5177 & 0.7755 & 0.8194 \\
\hline IBSR_02 & 0.6600 & 0.8175 & 0.8578 & 0.6464 & 0.8106 & 0.8448 \\
\hline IBSR_03 & 0.6350 & 0.7957 & 0.7750 & 0.6056 & 0.7927 & 0.7779 \\
\hline IBSR_04 & 0.5213 & 0.7926 & 0.7805 & 0.4570 & 0.7968 & 0.8370 \\
\hline IBSR_05 & 0.7094 & 0.8048 & 0.8269 & 0.7034 & 0.8160 & 0.8466 \\
\hline IBSR_06 & 0.7579 & 0.7057 & 0.7703 & 0.7469 & 0.7375 & 0.8077 \\
\hline IBSR_07 & 0.5634 & 0.7693 & 0.8750 & 0.5295 & 0.7655 & 0.9002 \\
\hline IBSR_08 & 0.6361 & 0.7651 & 0.8650 & 0.6140 & 0.7675 & 0.8866 \\
\hline IBSR_09 & 0.5585 & 0.7637 & 0.8873 & 0.5368 & 0.7550 & 0.8937 \\
\hline IBSR_10 & 0.6230 & 0.7812 & 0.8874 & 0.6026 & 0.7765 & 0.8995 \\
\hline IBSR_11 & 0.5286 & 0.7481 & 0.8720 & 0.5188 & 0.7477 & 0.8801 \\
\hline IBSR_12 & 0.5951 & 0.7635 & 0.8465 & 0.5796 & 0.7717 & 0.8738 \\
\hline IBSR_13 & 0.5530 & 0.7952 & 0.7822 & 0.5099 & 0.7998 & 0.8209 \\
\hline IBSR_14 & 0.6839 & 0.8501 & 0.8492 & 0.6540 & 0.8521 & 0.8656 \\
\hline IBSR_15 & 0.4869 & 0.8008 & 0.8466 & 0.4693 & 0.8020 & 0.8614 \\
\hline IBSR_16 & 0.4932 & 0.8068 & 0.8372 & 0.4603 & 0.7948 & 0.8410 \\
\hline IBSR_17 & 0.4949 & 0.8051 & 0.8314 & 0.4755 & 0.8165 & 0.8581 \\
\hline IBSR_18 & 0.4992 & 0.8036 & 0.8516 & 0.4598 & 0.7899 & 0.8705 \\
\hline Mean & $\mathbf{0 . 5 8 4 9 2}$ & $\mathbf{0 . 7 8 4 8}$ & $\mathbf{0 . 8 3 5 5}$ & $\mathbf{0 . 5 6 0 4}$ & $\mathbf{0 . 7 8 7 1}$ & $\mathbf{0 . 8 5 4 7}$ \\
\hline
\end{tabular}

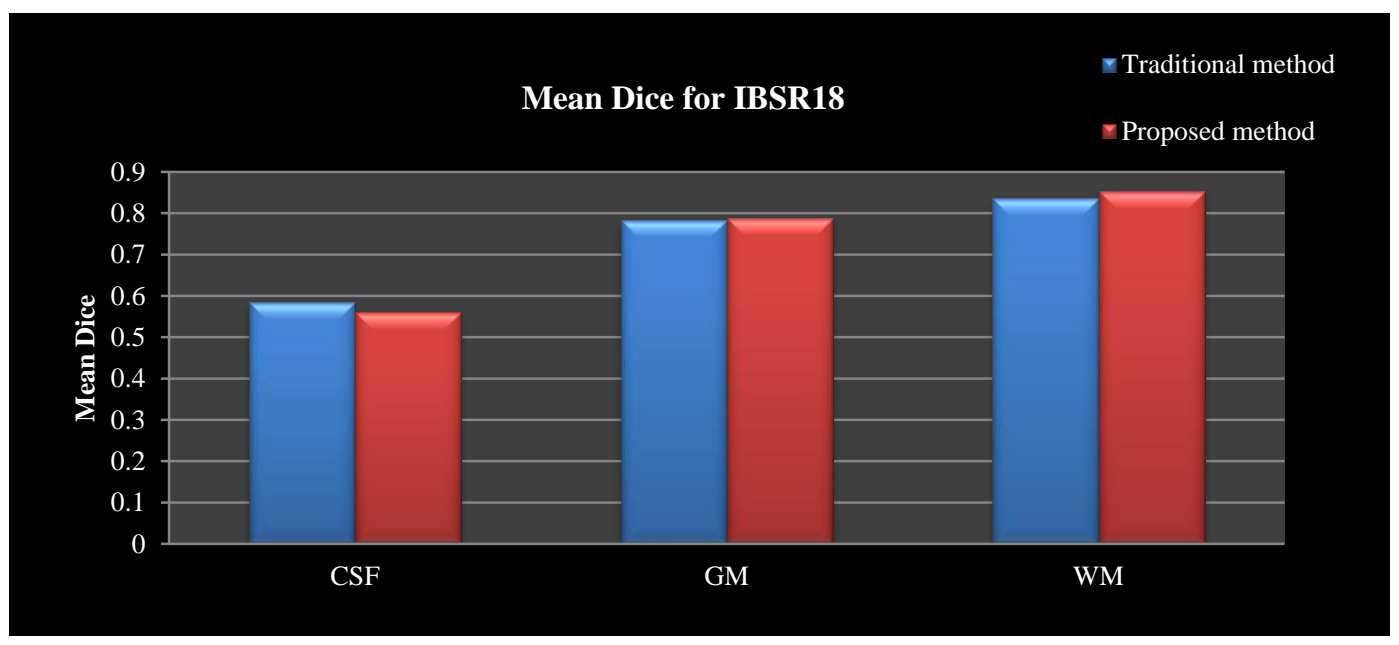

Fig.8. Mean Dice comparison between traditional and proposed HBSIKM for IBSR18 dataset 

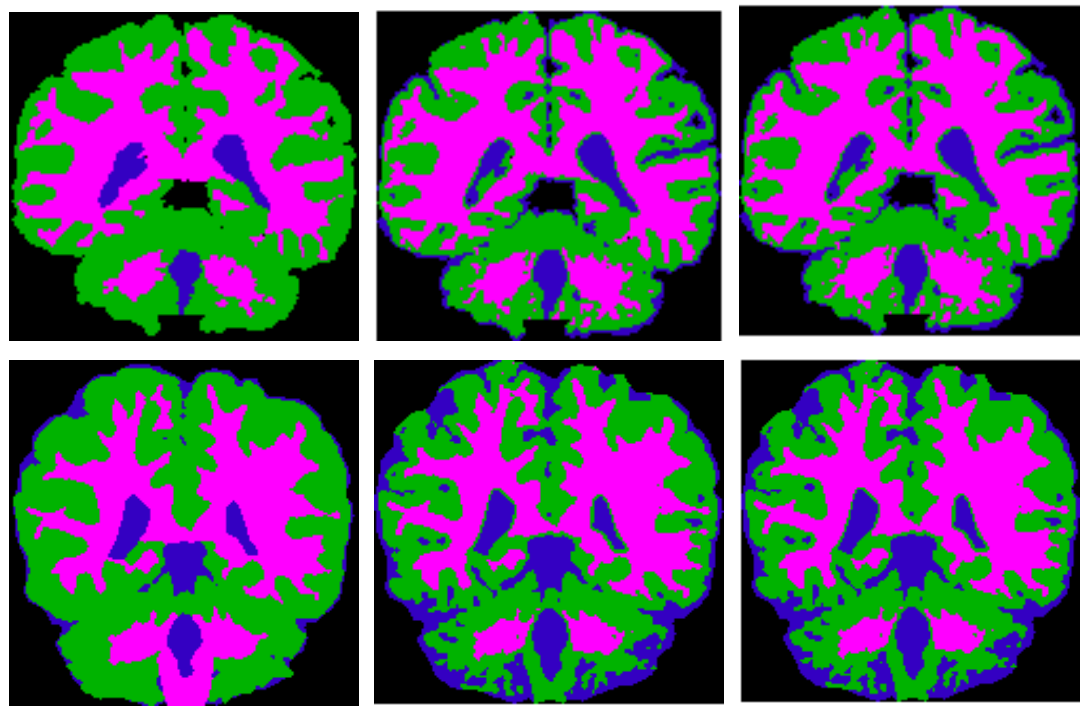

Fig.9. Segmented portions of CSF in blue color, GM in green color, WM in pink color. Column 1: Ground truth image,

Column 2: Results of the traditional method and Column 3: Results of the proposed HBSIKM method

The colored combination of CSF,GM and WM of ground truth, traditional $\mathrm{K}-\mathrm{Means}$ results and proposed HBSIKM method results are shown in Fig. 9 in columnwise respectively for the qualitative level comparision.

The proposed HBSIKM method shows better results than the traditional K-Means segmentation. Further our proposed method is faster than the traditional method. If the data volume is affected by any artifacts then the initialization process by the middle slice yields wrong results sometimes. This is considered to be a limitation of the proposed work. In future, a preprocessing related to artifact removal will be considered to prevent this overhead.

\section{CONCLUSION}

In this paper a histogram based self-initializing $\mathrm{K}$ Means segmentation algorithm (HBSIKM) has been proposed that initializes the K-Means technique automatically by the knowledge of MRI images and histogram processes. The experiments were carried out on 20 and $18 \mathrm{~T} 1$ coronal IBSR datasets. The results show that the proposed method speeds up the processing by reducing the number of iterations for each slice. Further the similarity measures of the proposed method shows better results than the traditional K-Means method

\section{REFERENCES}

[1] L. Zhong-Wei, N. Ming-Jiu, P. Zhen-Kuan, "Variation level set method for multiphase image classification", International Journal of Image, Graphics and signal Processing, MECS, 5: 51-57, 2011.

[2] R. Kandwal, A. Kumar, S. Bhargava, "Review: Existing Image Segmentation Techniques", International Journal of Advances Research in Computer Science \& Software Engineering, 4(4): 153-156, 2014.

[3] Yevgeniy Bodyanskiy, Olena Vynokurova, Volodymyr Savvo, Tatiana Tverdokhlib and Pavlo Mulesa, Hybrid Clustering-Classification Neural Network in the Medical
Diagnostics of the Reactive Arthritis, International Journal of Intelligent Systems and Applications, MECS, 8: 1-9, 2016

[4] Rabiu O. Isah, Aliyu D. Usman and A. M. S. Tekanyi Medical Image Segmentation through Bat-Active Contour Algorithm, International Journal of Intelligent Systems and Applications, MECS, 1: 30-36, 2017

[5] T. Kalaiselvi, K. Somasundaram, "Knowledge Based Self Initializing FCM Algorithms for Fast Segmentation of Brain Tissues in Magnetic Resonance Image", International Journal of Computer Applications, 90(14):19-26, 2014.

[6] T. Kalaiselvi, "Brain Portion Extraction and Brain Abnormality Detection from Magnetic Resonance Imaging of Human Head Scans", Pallavi Publications, India, 2011.

[7] A. R. Malali, J. Light, T. Kalaiselvi, X. Li, "Fall Pattern Classification from Brain Signals using Machine Learning Models", Journal of Selected Area in Telecommunications (JSAT), 3(10):1-5, 2015.

[8] A. Anamika, "Study of Techniques used for Medical Image Segmentation and computation of Statistical test for region Classification of brain MRI", I.J. Information Technology and Computer Science, MECS, 5: 44-53, 2013.

[9] L. Kaufman, P. J. Rousseeuw, "Finding Groups in Data, An Introduction to Cluster Analysis", John Wiley \& Sons, Inc., Canada, 1990.

[10] J. M. Pena, J. A Lozano, P. Larranaga, "An Empirical Comparisoin of Four Initialization Methods for the KMeans Algorithm", Pattern Recognition Letters, 20(10), 1027-1040, 1999.

[11] B. B. Bhusare, S.M. Bansode, "Centroids Initialization for K-Means Clustering using Improved Pillar Algorithm", International Journal of Advanced Research in Computer Engineering \& Technology, 3(4): 1317-1322, 2014.

[12] M. Tian, Q. Yang, A. Maier, I. Schasiepen, N. Maass, M. Elter, "An Automatic Histogram-Based Initializing Algorithm for K-means Clustering in CT", Bildverabeitung fur die medizin, Informatic Aktuell, Springer- Verlag Berlin Heidelberg, 2013.

[13] A. Mohamed, A. Wesam, "Efficient Data Clustering Algorithms: Improvements over K-means", I.J. Intelligent Systems and Applications, MECS, 3: 37-49, 2013. 
[14] T Raed. Aldahdooh, "DIMK-means 'Distance based initialization method for K-means clustering algorithm", I.J. Intelligent Systems and Applications, MECS, 2: 41-51, 2013.

[15] K. Somasundaram, S. Vijayalakshmi, T. Kalaiselvi, "Segmentation of Brain portion from MRI of head scans using K-means Cluster", International Journal of Computational Intelligence and Informatics, 1:75-79, 2011.

\section{Authors' Profiles}

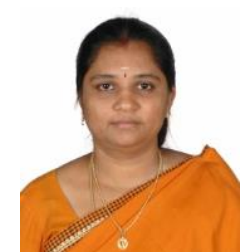

T. Kalaiselvi is currently working as an Assistant Professor in Department of Computer Science and Applications, The Gandhigram Rural Institute, Dindigul, Tamilnadu, India. She received her Bachelor of Science (B.Sc) degree in Mathematics and Physics in 1994 \& Master of Computer Applications (M.C.A) degree in 1997 from Avinashilingam University, Coimbatore, Tamilnadu, India. She received her $\mathrm{Ph} . \mathrm{D}$ degree from The Gandhigram Rural University in February 2010. She has completed a DST sponsored project under Young Scientist Scheme. She was a PDF in the same department during 2010-2011. An Android based application developed based on her research work has won First Position in National Student Research Convention, ANVESHAN-2013, organized by Association of Indian Universities (AUI), New Delhi, under Health Sciences Category. Her research focuses on MRI of human Brain Image Analysis to enrich the Computer Aided Diagnostic process, Telemedicine and Teleradiology Technologies.

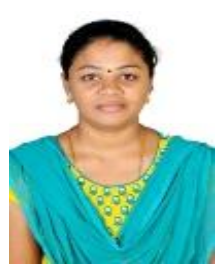

N. Kalaichelvi received her Bachelor of Sciences (B.Sc.) degree in Physics in 2007 and Master of Computer Science \& Applications in 2010 from The Gandhigram Rural University, Dindigul, Tamilnadu, India. She received her Master of Philosophy (M.Phil) degree in Computer Science in 2013 from Madurai Kamaraj University, Madurai, Tamilnadu, India. She was working as Assistant Professor from July 2010 - May2012 and from July 2015 March2016 in the Centre for Geoinformatics, Department of Rural Development, The Gandhigram Rural Institute - Deemed University, Dindigul, Tamilnadu, India. She was working as Assistant Professor from June - 2014 to June -2015 in the Department of computer Science in Prince Shri Venkateshwara Arts and Science College, Gowrivakkam, Chennai, Tamil nadu, India. Currently she is pursuing Ph.D. degree in The Gandhigram Rural Institute - Deemed University. Her research focuses on Brain Signal Segmentation. She has qualified State Eligibility Test for Lectureship in Feb-2016.

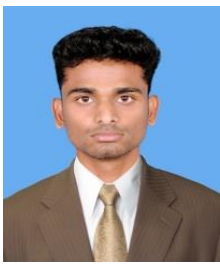

P. Sriramakrishnan received his Bachelor of Sciences (B.Sc) degree in Computer Science in 2011 from Bharathidasan University, Trichy, Tamilnadu, India. He received Master of Computer Science and Applications (M.C.A) degree in 2014 from The Gandhigram Rural Institute-Deemed University, Dindigul, Tamilnadu, India. He has worked as Software Developer in Dhvani Research and
Development Pvt. Ltd, Indian Institute of Technology Madras Research Park, Chennai during May 2014 - March 2015. He is currently pursuing Ph.D. degree in The Gandhigram Rural Institute - Deemed University. His research focuses on Medical Image Processing and Parallel Computing. He has qualified UGC-NET for Lectureship in June 2015.

How to cite this paper: Kalaiselvi $\mathrm{T}$, Kalaichelvi $\mathrm{N}$, Sriramakrishnan P, "Automatic Brain Tissues Segmentation based on Self Initializing K-Means Clustering Technique", International Journal of Intelligent Systems and Applications(IJISA), Vol.9, No.11, pp.52-61, 2017. DOI: 10.5815/ijisa.2017.11.07 